\title{
Supplemental Regulatory Submission
}

National Cancer Institute

\section{Source}

National Cancer Institute. Supplemental Regulatory Submission. NCI Thesaurus. Code C70862.

Additional documentary support presented to a regulatory agency to bolster a previous submission. 